\title{
An Analysis on the Adaptability of the People's Livelihood Policies
}

\author{
Fangmei $\mathrm{Yu}$ \\ Public Administration School of Hohai University \\ Nanjing, China \\ yufangmei1984@126.com
}

\author{
Guoqing Shi \\ Public Administration School of Hohai University \\ Nanjing, China \\ gshi@hhu.edu.cn
}

\begin{abstract}
The adaptability of the people's livelihood policies is a prerequisite to achieve policy objectives. To analyze the adaptability, it is not only to explore the coordination between the policy and the policy environment, but also to judge mutual adaptability between it with various stakeholders, policy implementation mechanism and the existing policies. For the environmental factors of people's livelihood policies, which are classified, and associated with specific contents of the policies in certain field, they need to be distinguished and considered.
\end{abstract}

Keywords-people's livelihood policies; adaptability; policy environment; stakeholders

\section{INTRODUCTION}

The goals are the soul of a policy. From a subjective point of view, there is no pointless policy. Policy objectives come true, the premise is that the policy must be adaptive, that is, the relationship between policy and environmental is harmonious and stable, showing a positive interaction state, the bias to any party is not sufficient to ensure to achieve the policy objectives.

Adaptability, originated in Darwin's evolution theory "survival of the fittest", has been used in the field of social sciences. In China, some researchers carried out studies on policy science and public policy by using methods and concepts of ecology. Cao Tangzhe defined the adaptability of public policy as: the public policy system interacts with public policy environment, and adapt to each other, to ensure public policy objectives come true, at the same time, to improve the public policy environment. From his analysis as well as theoretical origin point of view, the public policy environment is largely focused on its external environment. But for a policy, it also shall adapt to its internal environment - policy implementation mechanisms and other existing policies, in order to be successfully and effectively implemented. Therefore, Zhou Xinwei \& Lu Shuaibing defined it as: a mutually beneficial and virtuous cycle mode is formatted from that the public policy system interacts with policy environment, policy implementation mechanisms, and other policies. A successful people's livelihood policies are made to respond to the public and social needs, shall be accepted by the public. The policy implementation ties up with local governments and has to be supported by them, and the comprehensive qualities of executive determine the of policy performance. So, the adaptability of the people's livelihood policy not only involve the above mentioned, but also include a virtuous cycle mode between it with various stakeholders.

\section{CONSTRCUT A SYSTEM TO ANALYZE THE ADAPTABILITY OF THE PEOPLE'S LIVELIHOOD POLICIES}

\section{A. Adaptability to Stakeholders}

A livelihood policy will always distribute and adjust to the interests of some people, and will always constrain or change some people's behavior. Only when various stakeholders and the policy adapt to each other, it may have been accepted, implemented and enforced smoothly; its goals to be reached successful. Generally, the key stakeholders of public policy involve target groups, local governments and policy-performers.

1) Adaptability to target groups:First, it's needed to be examined the acceptability of the target groups who are affected by a people's livelihood policy. The livelihood policies are made and implemented to respond to public demands and to solve public problems. Only target groups can adopt it, public problems could be solved and the policy objectives could be reached. Because, accept, then support and obey, to promote policy goals; do not accept, may take an uncooperative attitude or passive resistances, and even take anti-policy measures, to resist the reform of the governments. Therefore, for livelihood policies, the attitudes, recognition, acceptance, supports and cooperation of the target groups will affect their expected effect to be generated.

Just as the impacts of the policy on each target group are different, so is the degree of recognition of them. The livelihood policy to meet the needs of target groups for interest, is the key factors affecting they accept it or not. For beneficiary groups, if a fee (tax) would is required for policy execution, their willingness and abilities to pay are important in the analysis on the adaptability between them with the policy; for groups that could be damaged, the policy execution may bring in some damages on their interests of society, economy, resources, environment and other aspects. What're their attitudes or appeal toward the policy? During policy making and implementation, some effective measures should be taken to eliminate or mitigate adverse effects.

Furthermore, the degree of political socialization of target groups, also can affect the policy implementation. The deeper the degree of political socialization of target groups is, the more likely it is to establish the belief that uphold, support and understand the existing political system and political authority, the easier policy implementation will be, and vice versa. 
2) Adaptability to local governments:Generally, the people's livelihood policies are implemented and expanded by local governments and relevant departments, the premise is that they can accept the policy, and give enough supports and cooperation to policy making and implementation. If disagree the policy, put the policy on the shelf, the policy would be empty, no role to play; and vice versa. So, the analysis on the policy adaptability to the local governments, will mainly consider their attitudes toward a policy, their acceptability, which supports and cooperation they will give to ensure the realization of the policy objectives, whether the appropriate measures can be taken to improve it.

3) Adaptability to policy executors: In an analysis on the adaptability between a livelihood policy and policy executors, main attention shall be put on whether the comprehensive qualities of policy executors meet the requirements to well carry out the policies. The executors' comprehensive qualities involve policy level, knowledge structure, ability structure, and working attitude, which reflect their abilities and levels of mastering, understanding and interpreting policy, operational level and capacity, work attitude, sense of responsibility and the dedication to work, etc. If their policy levels are limited, they couldn't correctly understand policy spirit; if operational level and capacity is not strong, it is difficult to effectively plan and organize activities of policy implementation, policy execution will be blocked; if their will is not firm enough to withstand setbacks or external environment of "sugar-coated bullets", the livelihood policy implementation would be given up halfway, or it is happened that the alienation of public power, corruption and bribery, the interests of the public would be damaged.

\section{B. Adaptability to Environment}

Whether a specific policy or policy group, its formulation and implementation are inseparable from a certain environment, and will make the corresponding change with the change in the environment. Here, from natural environment, social environment and international environment three aspects, a framework will be constructed to analysis the adaptability of people's livelihood policy.

1) Adaptability to natural environment: Natural environment is the material basis of existence and development of a state and nation, the foothold of socioeconomic construction, also the foundation of human social system, so it constitutes the basic environment of the livelihood policy system. during people's livelihood policy making and implementing, attention must be paid to analyze whether the policy is in line with local natural environment or not, whether policy design will beyond the carrying capacity of natural environment or not, whether its enforcement can cause damage on the natural environment or not.
2) Adaptability to social environment:Each public policy is rooted in a specific social environment; leaving the environment, it will be difficult to play its function and role, so are the livelihood policies. A policy to be executed successfully, it must be able to adapt to the local social environment, or reached a mutual balance, only in this way, the policy will be possible to achieve the desired effects. The social environment includes socio-economic status, political status, cultural status, legal status, population condition, among which the more important is socioeconomic status, political status, cultural status; they have more important and more direct influence, and restrictive or even decisive function. Economic strength affect the efficiency of national basic policy and its implementation, constitute the basic material conditions and economic environment of public policy.

a) Adaptability to political and legal environment:Political environment refers to the political situation of a country or a region in a certain period, including: political system, political situation, the degree of democratization, party politics, etc.

- Political environment factors determine the political nature of the people's livelihood policy.

- These factors determine the democratization extent of the people's livelihood policy.

- The political and legal environment factors determine the legalization degree of the people's livelihood policy.

- Political environment factors determine the operation condition of the people's livelihood policy.

- Political environment factors determine the social effect of people's livelihood policy.

There is a dialectical and unified relationship between political and legal environment and the people's livelihood policy. On the one hand, policy making and implementation rely on certain political and legal environment; on the other hand, the corresponding livelihood policies can solve relevant social problems, then playing a role in optimizing the political and legal environment.

b) Adaptability to economic environment:Economic environment refers to the material resources and productivity status of a country or a region, including: economic system, economic development level, price level, the status of market development and perfection, etc. Economy is the material basis of human society, the execution of a people's livelihood policy requires substantial fiscal funds. Thus, the economic environment is the primary consideration during making and implementing livelihood policies, and the decisions on people's livelihood must be consistent with the local socio-economic development, so that the policy can exist and develop.

- The economic environmental factors are the basic starting point of making and implementing livelihood policies. Resources distribution and 
existing stock, and the established economic system, are the foundation of the policy process.

- Economic environmental factors are necessary for the run of a people's livelihood policy. The policy process has to take certain human, material and financial resources, and requires certain economic system as a support. The policy performance, operation conditions and target realization degree, depend on the amount of resources and the quality of economic conditions.

- Economic environment factors can affect the objectives, contents and direction of people's livelihood policy. only the policy it is developed and implemented in accordance with national or local resources, and socio-economic conditions, it may be appropriate, its contents is likely to have the feasibility, its goals can be achieved. Thus, the real situation, the actual economic systems and structures, are restricting the policy objectives, contents and direction.

As can be seen, as resource conditions of a public policy, the economic environment determines and supports the people's livelihood policy's process, quality, operating conditions, the feasibility and direction of goals and contents. Therefore, to make decisions for the people's livelihood, the first considered factor is the status of the national or regional socio-economic development, to analyze and forecast whether the policy contents and objectives are in line with national or local socio-economic strength and development level or not; it will be likely to result in the failure that any decision is beyond or behind the national or regional economic strength and development levels. Because, if beyond, even if the policy goals are very good, however, due to the lack of policy resources, there will be only an illusion for policy implementation; eventually, it's difficult to realize the policy goals. If behind, the policies neither play due role in adjustment and allocation or reallocation, nor solve the problems on people's livelihood; even the governments might leave a bad impression on the public, such as "do not care, perfunctory, administrative omission".

c) Adaptability to humanistic environment:Humanistic environment includes two aspects: one is the levels of education and cultural of the nation or the region; the second is the social and cultural environment, mainly refers to the formation and changes of local social stratum structure, family structure, social customs and habits, beliefs and values, behavior norms, lifestyles, cultural traditions, population size and geographical distribution, etc. The impacts of humanities environment on the people's livelihood policy are as follows.

- Humanities environment factors determine the intellectual and technical conditions for operating the people's livelihood policy. In a society, where the education, culture, science and technology are relatively developed, the government can provide run in the people's livelihood policy of each link, with high-quality personnel, various kinds of up-todate technology and technique, and prepare comprehensive information as much as detail as possible.

- Humanities environment factors affect the moral, ethical and psychological conditions of running the people's livelihood policies. If a society has a tradition of paying attention to ethics and morals, atmosphere good, orderly; policy makers have a sense of justice, policy executors have a sense of responsibility, policy target groups have good psychological quality; the policy formulated not only is fair and reasonable, but also will be smoothly carry out.

- The cultural environment factors affect the value targets of a people's livelihood policy. If its value targets are contrary to China's traditional culture and moral culture, it is difficult for target groups to accept it, and to carry out it, and vice versa.

- Humanities environment factors provide social structure for the run of the livelihood policies. Social stratum structure, population structure and family structure are social phenomenon, vary along with the social productive forces, related to the culture environment of a society, and uncontrolled by subjective will. Whether it is to promote or hinder the operation and development of the policy, the designers can only comply with it, and through adjusting the policy design to cope with the challenges from the social structure. If the policy cannot meet the change of social structure, its effect will be reduced greatly.

3) Adaptability to international environment: International environmental factors include international pattern, the comprehensive national strength, international status, and international trend, etc. Globalization, marketization and informatization have expanded the scope of public policy; some transnational issues become the object of public policy; marketization makes the scope and function of public policy have been changed; following informatization, more and more public policies are under the supervision of the international society and world opinions; existence and the role of international organizations make the nation state partly lost their decision-making authority in some policy areas; international rules and regulations, resolutions and agreements gradually became the basis of many policies of many countries; even some international organizations and multinational corporations directly or indirectly participate in making and implementation of national and regional public policy. Here, it is very important that a country's international status, because it shows her position in international affairs, and also reflects a country's comprehensive national power. All these factors restrict and affect the national public policies. Therefore, for the national macro policies on people's livelihood, the formulation and implementation, sometimes are referring and learning experience of other countries or international organizations. Especially in China's accession to the WTO 
and to the world, it needs to be considered that whether the basic value idea of livelihood policy is convergent with international universal ideology, is consistent with the development trend of the world, adopts to the national comprehensive strength or not; whether the choice of its goals and value can safeguard national security and national interest or not.

\section{Adaptability to Other Policies}

Any public policy, to a certain extent, can only solve some policy issues within limited scope. To ultimately resolve the issues, it's often required that a mutual and systematic cooperation among a number of policies.

1) Whether the people's livelihood policy is in conflict with current policies or not:In reality, since the problems of functions, powers and responsibilities configuration, the same social problem could be managed by multiple government departments. In the policy process, policy subjects are diversifying. There are many policies and documents, sometimes which are disconnected, care for this and lose that; or inconsistent with other sectoral policies and documents; or in conflict with regulations; or lack of consistency or coordination among the policies at the upper and lower levels. But, people's livelihood policies are related to all aspects of social life, if the policies conflict with each other, the public will be at sea. In addition, if the policy conflicts stem from the strife of the government departments, it will also lead to a "policy vacuum" phenomenon, unluckily, no department takes the initiative to make a policy to solve a social problem; or the soil and space would be created for policy rent-seeking and corruption. Therefore, in livelihood policy making, adjusting or changing process, it is to carefully consider the coordination and adaptability of the policy with other public policies, so that it can play a proper role and really solve the social problems.

2) Whether there are supporting policies and engage with relevant public policies or not:To implement a new deal, sometimes must be accompanied by supporting policies and measures in order to successfully achieve the policy objectives. For the livelihood issues, all sorts of problems are implicated, if only solve one of them, the policy effect is difficult to appear, and the seriousness of other problems may be offset the policy effect. Thus, it is required that a corresponding supporting policies to achieve policy objectives.

Secondly, even homer sometimes nods. Even if a policy would be designed perfectly and considered comprehensively, in the face of the complicated social problems and the difficulty of getting accurate and complete information, in the process of policy implementation, it needs perfecting, interpreting, adding, updating, adjusting and other relevant activities.

Thirdly, policy implementation is a systematic project, needs equipping with corresponding human, financial and material resources, which have to be stipulated or supported by administrative system through relevant policies, documents and even laws and regulations.

Fourthly, the people's livelihood policies are to meet the social needs, when the people's low-level needs are met, their demand levels will rise, whether the policies engage with each other or not? Whether there will be policy fault or not? If the policies are not matching or connected, on the one hand, it would be difficult to carry out the policies; on the other hand, it would give policy executors an opportunity to find countermeasures or exploit loophole, which are likely to affect the policy social effects. So, these issues have to be considered carefully in people's livelihood policy process.

\section{Adaptability to Enforcement Mechanism}

An American scholar declared: in the process of achieve the goal of the U.S. government, the program only determines $10 \%$ of the function, while the remaining $90 \%$ is dependent on effective enforcement (or implementation). It shows how important the policy enforcement. If the policy enforcement mechanism is scientific and reasonable, it would be effectively enforced. What is policy implementation mechanism? As the name implies, it refers to the internal mode of action and linkages of a series of related elements with functional structure in the actual implementation process of a policy. It is the security to reach the decisions, and affects the extent of the achievement of policy objectives. After a policy is approved, if there is no corresponding enforcement mechanism, it not only will affect the policy effects, but also may lead to the failure of the policy, and even trigger social instability and disharmony. The adaptability between a people's livelihood policy and its enforcement mechanism can be analyzed from the following aspects.

1) Accrual allocation mechanism:Generally, the people's livelihood policies are set and executed by government agencies, only the powers and responsibilities (particularly the fiscal commitment) are reciprocal, local governments and relevant institutions will effectively implement the policies. Thus, a reasonable accrual allocation is a precondition for the effective implementation of policies.

First, vertically, the allocation of power and purse strings at different levels of governments and departments should be reasonable. In China, public financial administration system is four levels: the centre, provinces (autonomous regions and municipalities directly under the central government), cities (prefectures), and counties. The execution and implementation of a people's livelihood policy requires substantial fiscal support, as the powers are decentralized, and the finance is turned over to the higher authorities, the powers and purse strings of local governments don't match. during executing people's livelihood policy, if local governments and organizations at a lower level take too many responsibilities, under fiscal pressures, they may decrease the policy implementation force, and reduce the welfare level of the target group. In addition, fiscal pressures may also affect the stability and continuity of the policies. 
Second, horizontally, the allocation of power and responsibilities for different local governments and functional departments in different areas should be clear, balanced and coordinate. (a) The accrual allocation for different local governments should be fair. Otherwise, it will affect the enthusiasm of local governments to carry out the policy and the realization of the policy goals. (b) The executive power allocation for different departments should be clear and coordinate, because the formulation and execution of people's livelihood policies need multiple functional departments to cooperate.

2) Policy redefinition mechanism:In China, in the system context of "isomorphic responsibility" and "progressive contract", almost all of the important policies are set by the central government, so are the people's livelihood policies. In order to make a policy to adapt to each province, autonomous region and municipality directly under the central government with quite different socioeconomic conditions, the central policies often tend to be more macro and general. Local governments, in light of local actual situation, develop a more specific or detailed policy implementation plans or methods, on the one hand, to pass policy, decompose policy and develop policy, on the other hand, to organize and carry out the policy and supervise policy process. Actually, these processes are also the processes that local governments redefine and adjust the policy according to local situations, which includes not only the normal, reasonable and flexible disposal, but also unreasonable modifications. Lin Mei summarizes four kinds of common unreasonable policy redefinition ways: "loopholes", "distorting the rules" "adding more documents", "make only superficial changes". Which in part result from incomplete information or incomplete policy, local interests and egoism; lead the policy goal to deviate from the original center. Because, "there is always a countermeasure to cope with the government policies", the public doubt local governments, "policies are changed at local", which make the government's credibility and image damaged. Therefore, during the people's livelihood policy implementation that is concerned by the public, if the "Deviation" can be kept in a reasonable range during policy definition, the people's livelihood policy could achieve the desired effects and reach its goals.

3) Information disclosure, communication and feedback mechanisms:Information is not only the important basis of making people's livelihood policies, but also the important guarantee to smoothly implementing or enforcing the policies, and the source of policy assessment.

First, information disclosure is to protect the public's right to know, to enhance the transparency of government work, and is the foundation of smooth enforcement of the policies. It can improve the awareness and transparency of a policy, and let the public to understand policy goals and contents, then obtaining recognition and support of policy object; Conversely, the difficulty and risk of policy implementation will increase.

Second, the smooth flow of information channels and sources, are the assurance of timeliness and completeness of information, and necessary for the effective policy execution. As long as the communication mechanisms are sound, the idea and information exchanges among various departments at different levels and personnel will be smooth, to promote mutual understanding, to enhance collaboration, cohesion, integration force and the driving force of the system, then achieving support and cooperation to improve the overall effect of policy implementation.

Third, a perfect information feedback mechanism, is helpful in getting to know the policy execution, correcting the implementation deviation, ensuring the effects of policy implementation, and is also a reliable source of policy evaluation.

4) Public participation mechanism:Democratic participation is an important channel that realizes citizen involvement in national policy in accordance with the laws, and the public really enjoy the rights of managing the state and public affairs; is also the fundamental measure to reflect public sentiments and opinions, and improve the level of democracy in policy implementation. For public participation in policy implementation, however, there are some problems, such as the shortcomings of participators, the conflict between the governments and the public, and the non-institutional factors in public participation. While the livelihood policies involve all aspects of life of target groups, benefits will drive them to participate in policy enforcement. So, it has to consider that, for the public abilities, and the participation capacity, channels, content, methods, scope, procedures, and guarantee mechanism, etc., whether adapt to or ensure policy effective execution or not.

5) Supervision mechanism:Control is an important part of management and an important means of policy implementation. Adopted a strict policy monitoring, to ensure policy scientific formulation and effective implementation, the implementation deviation can be found and corrected in time and. Monitoring results can become the basis of job evaluation and rewards and punishment. Here, therefore, in the people's livelihood policy implementation process, it need to analyze that, for the existing monitoring mechanism, organizations and their status, system arrangement and the procedures, whether can play their role to ensure or promote policy effective implementation or not. If not, analyze the reasons.

From the results of the analysis, it is not hard to see, if the above mechanisms are imperfect, the situation of achievement of policy goals is an open question. If people's livelihood policies should play their proper functions and effects, there must be a set of corresponding policy implementation mechanisms. 


\section{CONCLUSION}

Policy environment factors are classified, and certain categories of environmental factors are always associated with a policy in certain field and with specific contents. So, during formulating and implementing specific policies, it will be taken into consideration that a distinction should be made among all environmental factors, and the corresponding environmental factors should be selectively identified and evaluated. For example, during making the policies on education, science and technology, social cultural and psychological environment shall be considered. So, in this regard, the policy subject should think about the issues targeted as far as possible.

\section{ACKNOWLEDGMENT}

Supported by the National Social Science Foundation of China (Grant No. 07ASH010)

\section{REFERENCES}

[1] Cao Tangzhe. The Issues on the Adaptability of Government Public Policies after China Joint into WTO [J]. No.4, 2002, pp.111-116.

[2] Zhou Xinwei, Lu Shuaibing. An Analysis on the Adaptability of Public Policies [J]. Journal of Hunan Agricultural University (Social Sciences), Vol.9, No.5, 2008, pp.99-102.

[3] Zhang Kaiping. On the Impacts of Social Environment on Policy Implementation [J]. Market Weekly (Disquisition Edition), No.12, 2008, pp. 145-146.

[4] Lin Mei. A Study on the Implementation Mechanism of the Environmental Policy: A frame of system analysis [J].Sociological Research, No.1, 2003, pp. 102-110.

[5] Wei Chunyan, Wang Lin. A Discussion on Public Particition in Public Policy Implementation [J].Theroy Monthly, No.1, 2009, pp. 68-70.

[6] Hu Ningsheng. An Analysis on Public Particition in Public Policy Implementation [J].Chinese Public Administration, No.12, 1999, pp.51-54. 